BULLETIN Bulletin hispanique

HISPANIQUE Université Michel de Montaigne Bordeaux

$115-2$ | 2013

Les traductions vieillissent-elles ?

\title{
Sur les Exercices de style de Raymond Queneau
}

\section{Aina López Montagut}

\section{OpenEdition}

Journals

Édition électronique

URL : https://journals.openedition.org/bulletinhispanique/2900

DOI : 10.4000/bulletinhispanique.2900

ISSN : 1775-3821

Éditeur

Presses universitaires de Bordeaux

Édition imprimée

Date de publication : 28 décembre 2013

Pagination : 697-711

ISBN : 978-2-86781-908-7

ISSN : 0007-4640

Référence électronique

Aina López Montagut, «Sur les Exercices de style de Raymond Queneau », Bulletin hispanique [En ligne], 115-2 | 2013, mis en ligne le 14 février 2017, consulté le 21 septembre 2021. URL : http://

journals.openedition.org/bulletinhispanique/2900; DOI : https://doi.org/10.4000/bulletinhispanique. 2900

Tous droits réservés 


\title{
Sur les Exercices de style de Raymond Queneau
}

\author{
Aina López Montagut \\ Université Paris-Sorbonne
}

L'ouvre de Raymond Queneau Exercices de style a été traduite en italien par Umberto Eco en 1983. Nous proposons dans l'article qui suit l'analyse de quelques variations d'intérêt plutôt linguistique traduites en italien, et parfois même nous les comparons avec d'autres langues dans le but de percevoir les solutions envisagées par les traducteurs.

Mots-clés: Raymond Queneau, Exercices de style, Umberto Eco, traduction.

Exercices de style de Raymond Queneau fuetraducido en italiano en 1983 por Umberto Eco. En este artículo se analizan algunos ejercicios de interés más bien lingüistico traducidos en italiano e incluso, en algunos casos, se comparan con otros idiomas para ver las soluciones traductológicas propuestas por los traductores.

Palabras clave: Raymond Queneau, Exercices de style, Umberto Eco, traducción.

"Exercices de Style", Raymond Queneau's work, was translated into Italian in 1983 by Umberto Eco. In this article we will study some linguistics exercises translated in Italian and for some of those, we will compare them with translations in other languages, in order to understand the solutions proposed by the translators.

Keywords: Raymond Queneau, Exercices de style, Umberto Eco, translation.

\section{LES DÉBUTS DES EXERCICES DE STYLES}

Raymond Queneau (1903-1976) a été journaliste, romancier, poète, essayiste, traducteur de l'anglais (Le mystère du train d'or d'Edgar Wallace en 1934, par exemple)... mais l'un de ses écrits l'a rendu tout particulièrement célèbre : il s'agit des Exercices de style (1947). 
Il semblerait que pour l'écriture des Exercices de style Queneau se soit inspiré de l'œuvre de Bach Die Kunst der Fuge (composée entre 1740 et 1750) qui comporte quatorze fugues et quatre canons. Chacun de ces dix-huit contrepoints se base sur le Contrapunktus 1. Queneau dit qu'après avoir entendu l'Art de la Fugue il a pensé " qu'il serait bien intéressant de faire quelque chose de ce genre sur le plan littéraire [...]. C'est effectivement et très consciemment en me souvenant de Bach que j'ai écrit Exercices de style »".

En mai 1942, Queneau compose les douze premiers exercices et les intitule Dodécaèdre, en raison de la figure géométrique à douze facettes. Ces douze exercices, qui sont les premiers du livre actuel, seront refusés en 1942.

La première édition des Exercices de style date de 1947 et a été publiée par Gallimard. Queneau dit : "Je m'arrêtai au quatre-vingt dix-neuvième, jugeant la quantité satisfaisante; ni trop, ni trop peu : l'idéal grec quoi $»^{2}$.

Quant au nombre de textes, Carol Sanders dit : « [...] au lieu de 99 histoires, il aurait pu y en avoir 999 ; d'ailleurs, dans l'édition de 1963, certains exercices sont remplacés par d'autres " ${ }^{3}$. Il ajoute qu' " après avoir montré dans ces 99 exercices que même l'incident le plus quotidien est d'une richesse presque inépuisable, Queneau semble inviter le lecteur à prendre part à l'effort de création et à remplir lui-même la centième case vide $»^{4}$.

En 1963, Gallimard publie une deuxième édition accompagnée de 99 «Exercices de style typographiques » de Robert Massin et de 45 « Exercices de style parallèles peints, dessinés ou sculptés » de Jacques Carelman.

Le typographe et l'illustrateur proposent une mise en page unique pour chaque variation. On remarque en consultant cette édition que Massin joue parfois sur la présentation du texte comme pour l'illustration de Passé indéfini, Présent, Prétérit, Imparfait. En effet, dans ce cas quatre textes qui étaient présentés séparément dans l'œuvre de Queneau se retrouvent sur une même partition. Chaque temps verbal a sa portée et comme dans une vraie partition, les quatre portées - ici les quatre temps - forment un système. Dans chaque système on peut voir une unité de sens mais une diversité temporelle. Chaque action s'est produite dans un moment différent du temps mais est représentée dans le même système de la partition.

Dans le cas de Télégraphique, Massin joue là aussi sur l'apparence du texte mais plus précisément sur une représentation réelle d'une situation imaginaire. Dans d'autres situations, ne pouvant rien changer au texte, Massin illustre le récit à travers le titre comme c'est le cas pour Mathématique ou Paysan.

Carelman propose quant à lui des représentations plus artistiques en ce qu'une même situation devient un tableau, une sculpture, une photographie... On peut voir par exemple Mosä̈que, Tarot de Marseille et Ombres chinoises.

1. Raymond Queneau, Exercices de style accompagnés de 99 Exercices de style typographiques de Robert Massin et de 45 Exercices de style parallèles peints, dessinés ou sculptés de Jacques Carelman, Paris, Gallimard, 1963, p. 9.

2. Ibid., p. 10 .

3. Carol Sanders, Raymond Queneau, Amsterdam/Atlanta, Rodopi, 1994, pp. 91-92.

4. Ibid., p. 92. 
Une " nouvelle " édition est proposée en 1973. Dans chacune des éditions il y a 99 exercices : le premier s'intitule Notations et est suivi de 98 variations. Chacune des variations se base sur cette histoire réelle et banale qui commence dans un bus et se termine devant une gare pendant que deux hommes parlent d'un bouton mal placé. Cette histoire qui pourrait sembler sans intérêt deviendra pourtant la matrice de 98 jeux d'écriture et de stylistique :

Dans l'S, à une heure d'affluence. Un type dans les vingt-six ans, chapeau mou avec cordon remplaçant le ruban, cou trop long comme si on lui avait tiré dessus. Les gens descendent. Le type en question s'irrite contre un voisin. Il lui reproche de le bousculer chaque fois qu'il passe quelqu'un. Ton pleurnichard qui se veut méchant. Comme il voit une place libre, se précipite dessus.

Deux heures plus tard, je le rencontre Cour de Rome, devant la gare Saint-Lazare. Il est avec un camarade qui lui dit : "Tu devrais faire mettre un bouton supplémentaire à ton pardessus ». Il lui montre où (à l'échancrure) et pourquoi ${ }^{5}$.

\section{Carol Sanders écrit dans Raymond Queneau:}

Certains exercices sont regroupés thématiquement (les temps verbaux, les cinq sens), mais l'ensemble se transforme et se renouvelle sans cesse $[\ldots]$... un monde fascinant, peuplé de personnages dotés de professions variées (" Médical ", "Lettre Officielle »), doués de caractères bien marqués ("Précisions ", "Hésitations ", "Injurieux »), affublés de tics linguistiques (" Moi je ", "Alors ") et aux origines variées ("Anglicismes ", "Italianismes ", "Paysan » [...]) [...]. Ces exercices frôlent de près la caricature parce qu'ils veulent faire rire, mais ils contiennent aussi une bonne dose d'originalité qui stimule l'imagination du lecteur ${ }^{6}$.

C'est donc à partir de Notations que vont se décliner divers aspects de la langue et de la forme. Queneau joue avec la langue et crée parfois sa langue.

Francesco Ragusa écrit quant à lui dans Raymond Queneau attraverso gli esercizi di stile :

Se Queneau [...] rinnega un certo tipo di linguaggio, è semplicemente per sostituirlo con un altro, non per fare tabula rasa di tutto [...] Queneau ha una fiducia immensa nella potenzialità del linguaggio a condizione di rinunciare a una lingua ormai inutile per fare opera autentica di rinnovamento. [...] Potremmo persino pensare che gran parte dei romanzi o delle poesie di Queneau non rappresentino che il modo di fornire un contesto a quello che sarà poi il vero protagonista, il linguaggio, che tende così a diventare l'obiettivo di se stesso. Linguaggio quindi non tanto come strumento, quanto fine, messaggio non volto a descrivere $[. .$.$] ma a dichiarare { }^{7}$.

Ce nouveau langage proposé par Queneau devient donc un vrai défi pour tout traducteur. Certains exercices comme ceux ne proposant qu'un changement du temps verbal employé seront certes plutôt faciles à traduire.

5. Raymond Queneau, Esercizi di stile (version bilingue), Torino, Einaudi, 2008, p. 2.

6. Carol Sanders, op. cit., pp. 92-93.

7. Francesco Ragusa, Raymond Queneau attraverso gli esercizi di stile, Cosenza, Luigi Pellegrini Editore, 1988, pp. 25-26. 
Cependant d'autres exercices qui créent une nouvelle langue ou imitent un accent devront être transposés dans une nouvelle langue parallèle, proche de celle du traducteur et donc du lecteur cible.

\section{UMBERTO ECO TRADUCTEUR DES EXERCICES DE STYLE}

On prend comme texte source la version bilingue français-italien de l'œuvre de Raymond Queneau traduite en italien par Umberto Eco en $1983^{8}$. Umberto Eco présente dans son introduction son texte de base, bien que la traduction qu'il propose pour Notations soit un peu différente. Il écrit à propos de son texte de base : "per tutti i metaplasmi [...], nonché per la duplice operazione metatattica delle permutazioni per gruppi di lettere e di parole, ho lavorato su di un unico testo base [...] ").

Voici ce texte :

Un giorno verso mezzogiorno sopra la piattaforma posteriore di un autobus della linea $S$ vidi un giovane dal collo troppo lungo che portava un cappello circondato d'una cordicella intrecciata. Egli tosto apostrofo il suo vicino pretendendo che costui faceva apposta a pestargli i piedi ad ogni fermata. Poi rapidamente egli abbandonò la discussione per gettarsi su di un posto libero. Lo rividi qualche ora più tardi davanti alla Gare SaintLazare in gran conversazione con un compagno che gli suggeriva di far risalire un poco il bottone del suo soprabito ${ }^{10}$.

Et voici ses Notazioni :

Sulla $S$, in un'ora di traffico. Un tipo di circa ventisei anni, cappello floscio con una cordicella al posto del nastro, collo troppo lungo, come se glielo avessero tirato. La gente scende. Il tizio in questione si arrabbia con un vicino. Gli rimprovera di spingerlo ogni volta che passa qualcuno. Tono lamentoso, con pretese di cattiveria. Non appena vede un posto libero, vi si butta. Due ore più tardi lo incontro alla Cour de Rome, davanti alla Gare Saint-Lazare. E' con un amico che gli dice: "Dovresti far mettere un bottone in più al soprabito". Gli fa vedere dove (alla sciancratura) e perche' ${ }^{11}$.

On remarque clairement que bien que les deux textes racontent d'une certaine façon la même histoire, ils se différencient à travers le récit.

Intéressons-nous maintenant au texte source sur lequel a travaillé Umberto Eco pour sa traduction. Entre l'édition de 1947 et l'actuelle on remarque quelques changements. Ainsi que l'indique Umberto Eco dans l'introduction qui précède sa traduction, cinq variations ont disparu dans la nouvelle édition : Permutations de 2 à 5 lettres, Permutations de 9 à 12 lettres, Hai Kai, Féminin et Réactionnaire.

8. Raymond Queneau, Esercizi di stile, op. cit.

9. Ibid., p. 11 de l'introduction.

10. Ibid., p. 11 de l'introduction.

11. Ibid., p. 2. 
En outre cinq autres exercices ont été rajoutés : Ensembliste, Définitionnel, Tanka, Translation et Lipogramme. Dans Définitionnel on remarque sans difficulté les définitions sous-entendues par le titre, en voici trois : «[...] grand véhicule automobile public de transport urbain $[\ldots],[\ldots]$ partie du corps qui joint la tête aux épaules [...] meuble disposé pour qu'on puisse s'y asseoir » respectivement pour le bus, le cou et le siège $»^{12}$.

En lisant Tanka on comprend l'essentiel de l'histoire ; ce qu'avait dit Queneau avec Notations est repris en résumé dans Tanka. On ne connaît pas les détails de la trame mais le contenu général se retrouve. Cette variation répond aux questions basiques permettant de comprendre une histoire : qui, quoi, quand et où... :

$\begin{array}{ll}\text { L'autobus arrive } & \text { Quoi ? } \\ \text { Un zazou à chapeau monte } & \text { Qui ? } \\ \text { Un heurt il y a } & \text { Quoi ? } \\ \text { Plus tard devant Saint-Lazare } & \text { Quand ? Où? } \\ \text { Il est question d'un bouton }{ }^{13} & \text { Quoi ? }\end{array}$

Par ailleurs, Queneau répond également aux possibles interrogations du lecteur quant au lieu, le temps ou l'action dans Analyse logique : "Plate-forme d'autobus. C'est le lieu. [...] Environ midi. C'est le temps. [...] Une querelle de voyageurs. C'est l'action $»^{14}$.

La variation Translation présente également un intérêt en ce que le traducteur pourrait éventuellement faire une double translation; soit garder les changements proposés par Queneau dans le texte cible, soit en proposer d'autres étant donné que Queneau déplace l'action dans un autre environnement. On peut relever certains exemples : " $S$ " devient " $Y$ ", " une heure d'affluence " " hexagone d'affouragement ", " un type " " un typhon ", le " cou " une " couchette " et un "voisin " un "voiturier $" 15$.

Enfin, certaines variations se retrouvent dans la nouvelle édition de 1973 mais sous un autre nom : Homéoptotes, Prétérit, Noble, Latin de cuisine, Mathématique et Permutations de 1 à 4 .

Selon Umberto Eco, les changements de titre sont dus à une préoccupation de justesse rhétorique.

En effet, dans le cas d'Homéoptotes qui est devenu Homéotéleutes, le changement apporte une justesse rhétorique en ce que le suffixe-ule n'est pas une marque d'homéoptote - structure syntaxique répétée - mais d'homéotéleute - son répété à la fin de plusieurs mots - .

Umberto Eco fait également état des difficultés rencontrées en tant que traducteur dans l'introduction qu'il présente au début de l'édition bilingue

\footnotetext{
12. Ibid., p. 156.

13. Ibid., p. 158.

14. Ibid., p. 52.

15. Ibid., p. 166.
} 
Pour Loucherbem, texte représentatif de l'argot des bouchers parisiens, Eco n'a pas voulu le traduire en dialecte puisqu'il avait déjà utilisé ce dernier pour d'autres variations. Le problème de la traduction des dialectes est qu'ils ne se correspondent pas. Loucherbem a donc été remplacé par Reazionario.

Dans le cas d'Homophonique, le traducteur l'a supprimé en choisissant une simplification linguistique et lexicale car « il francese è ricco di omofoni e l'italiano no $»^{16}$. Il a décidé de le remplacer par Vero?

En ce qui concerne Distinguo, le titre reste le même mais Eco dit qu'il a dû s'adapter à la langue d'arrivée : «è anch'esso basato su omofonie, e l'ho trasformato in un gioco di equivoci lessicali fondati su omonimie e omografie. Ogni lingua ha i suoi problemi $»^{17}$.

En voici un extrait :

Dans un autobus (qu'il ne faut pas prendre pour un autre obus), je vis (et pas avec une vis) un personnage (qui ne perd son âge) coiffé d'un chapeau (pas d'une peau de chat) cerné d'un fil tressé (et non de tril fessé). Il possédait (et non pot cédait) un long cou (et pas un loup con $)^{18}$.

Un bel di sul torpedone (non la torre col pedone) scorsi (ma non preteriti) un tipo (non un carattere a stampa) ovvero un giovinotto (che non era un sette da poco crecsiuto), munito (si, ma non scimunito) di un cappello incoronato (non incornato) da un gallone (non di birra), e con un lunghissimo collo (non postale) ${ }^{19}$.

\section{Présentation de Quelques variations traduites Par Umberto Eco}

Évoquons tout d'abord le cas de Lipogramme : Queneau n'en avait écrit qu'un qu'on pourrait appeler « lipogramme en e ". Ainsi ce texte s'illustre par l'absence de la deuxième voyelle sauf dans la présence de la conjonction de coordination « et " placée au centre du texte. "Et » s'avère être par conséquent l'exception qui s'ajoute au «e » du titre et se retrouve au milieu de l'axe séparant «voici » et " voilà ». Il suffit de regarder le texte pour remarquer cette symétrie parfaite entre « voici » et "voilà »"

Quelques années auparavant, en 1969, Georges Pérec avait écrit $L a$ Disparition où tout le texte était un lipogramme en " e ", ce qui aurait pu influencer Queneau. Il semblerait cependant qu'ils ne soient pas les premiers à priver leur langue d'une voyelle comme le dit l'homme de lettres Ugo Dotti dans "L'uomo che visse 99 volte ", dans L'Unità du 8 septembre 1983: "Nestore di Laranda, nel III o IV secolo, ha scritto un "Iliade" in cui la lettera "A" non compare mai in alcuna parola del I canto; Fulgenzio, nel VI secolo, ha fatto lo stesso $"^{21}$.

16. Ibid., p. 7 de l'introduction.

17. Ibid.

18. Ibid., p. 42.

19. Ibid., p. 43.

20. Ibid., p. 162.

21. Ugo Dotti, «L'uomo che visse 99 volte ", L'Unità du 8 septembre 1983, p. 9. 
Umberto Eco a quant à lui préféré faire une traduction plurielle, en ce qu'il part d'un seul lipogramme pour en faire cinq autres, un avec chaque voyelle. Ainsi, dans le premier « a mezzogiorno » et " autobus » deviennent " mezzogiorno " et "bus "22 (il manque des prépositions et des formes verbales telles que " ha " du verbe " avere »), dans le deuxième " diciamo $\|^{23}$ remplace un éventuel "verso ", dans le troisième " volta " ${ }^{24}$ remplace " giorno ", dans le quatrième la métaphore dessinée par " giraffa » ${ }^{25}$ cache « collo » et " dì » que l'on a par exemple en dialecte lombard remplace " giorno » et enfin dans la dernière version " non certo corto ${ }^{26}$ remplace «lungo".

$\mathrm{Si}$ l'on consulte la version allemande, on remarque que les deux traducteurs Ludwig Harig et Eugen Helmlé ont choisi de garder l'idée de Queneau, c'està-dire un lipogramme en " e ", ainsi que la symétrie entre " das war so " et «so war das".

Qu'en est-il de la version espagnole proposée par Antonio Fernández Ferrer? La voyelle " a » est la plus récurrente en espagnol donc le traducteur devrait faire un lipogramme en " a " en espagnol pour respecter la difficulté choisie par Queneau à travers son lipogramme en " e ». Cependant en espagnol on a la même situation qu'en français, un lipogramme en " e ". L'idée première de Raymond Queneau a été respectée, mais pas sa difficulté.

Venons-en au cas d'Alors :

Alors l'autobus est arrivé. Alors j'ai monté dedans. Alors j'ai vu un citoyen qui m’a saisi l'œil. Alors j'ai vu son long cou et j'ai vu la tresse qu'il y avait autour de son chapeau. Alors il s'est mis à pester contre son voisin qui lui marchait alors sur les pieds. Alors, il est allé s'asseoir.

Alors, plus tard, je l'ai revu Cour de Rome. Alors il était avec un copain. Alors, il lui disait, le copain: tu devrais faire mettre un autre bouton à ton pardessus. Alors. ${ }^{27}$

Cet exercice est tout particulièrement intéressant en ce qu'il a été traduit deux fois par Eco : Dunque, cioè et Vero. Dans le texte français, trois « alors " marquent une pause et annoncent le texte grâce à une virgule alors que le dernier suivi d'un point semble ne pas être à sa place. Cet " alors " final sert peut-être à dire qu'une suite est possible avec ce fonctionnement. Toutes les autres répétitions de l'adverbe "alors " se trouvent au début de chaque phrase sauf un seul qui signifie ici " pendant ce temps là ». " Alors " peut rappeler le mode de parler de l'enfant comme dans l'extrait "alors j'ai monté dedans". En effet en espagnol on a "entonces ", mais on aurait également pu avoir " y luego ".

22. Raymond Queneau, Esercizi di stile, op. cit., p. 163.

23. Ibid.

24. Ibid., p. 164.

25. Ibid.

26. Ibid.

27. Ibid., p. 84 . 
Dans les deux textes italiens, Eco semble quant à lui reproduire dans chaque texte d'arrivée un peu du texte source. En effet, le «alors» final que l'on retrouvait dans le texte de Queneau se retrouve avec le "Cioè » final d'Eco. En revanche, le jeu de la ponctuation se retrouve peut-être avec les points d'interrogation précédés d'un « vero "... Là où Queneau commençait les phrases avec « alors », Eco les termine avec « vero ? " ${ }^{28}$ :

Dunque, cioè, l'autobus è arrivato. Cioè ci sono montato; dunque, cioè, ho visto un tipo che mi ha colpito. Cioè, ho visto, dunque, quel collo lungo e la treccia intorno, dunque, al suo cappello. Cioè, dunque, lui si è messo a baccagliare col vicino che ciò̀ gli marciava sui ditoni. Cioè, dunque, lui è andato a sedersi.

Dunque, più tardi, cioè alla Gare Saint-Lazare, l'ho rivisto, dunque. Cioè, era con un tale che, dunque, gli diceva, cioè quel tale: "dunque, dovresti far mettere un altro bottone, dunque, al soprabito. Cioè.

L'autobus, vero, è arrivato, vero, e ci son salito, vero? Poi ho visto, vero, un tipo, vero, che mi ha molto colpito, vero, per il suo collo, vero, assai lungo, vero, e una treccia sul cappello, vero?

Lui si è messo, vero, a discutere, vero, con un vicino che gli pestava, vero, i piedi, vero? Poi è andato a sedersi, vero?

Più tardi, vero, l'ho rivisto, vero, alla Cour de Rome, vero, con un amico, vero? E questi, vero, gli diceva che avrebbe dovuto, vero, aggiungere, vero, un bottone, vero, al soprabito.

Venons-en maintenant au cas de l'exercice Précisions ${ }^{29}$ :

A $\mathbf{1 2 h 1 7}$ dans un autobus de la ligne $S$, long de 10 mètres, large de 2,1, haut de 3,5 , à $3 \mathrm{~km} 600$ de son point de départ, alors qu’il était chargé de 48 personnes, un individu du sexe masculin, âgé de 27 ans 3 mois 8 jours, taille $1 \mathrm{~m} 72$ et pesant $65 \mathrm{~kg}$ et portant sur la tête un chapeau haut de $\mathbf{1 7}$ centimètres dont la calotte était entourée d'un ruban long de $\mathbf{3 5}$ centimètres, interpelle un homme âgé de $\mathbf{4 8}$ ans, $\mathbf{4}$ mois 3 jours, taille $1 \mathrm{~m} 68$ et pesant $77 \mathrm{~kg}$, au moyen de 14 mots dont l'énonciation dura 5 secondes et qui faisaient allusion à des déplacements involontaires de $\mathbf{1 5}$ à 20 millimètres.

118 minutes plus tard, [...], taille $1 \mathrm{~m} 70$ et pesant $71 \mathrm{~kg}[\ldots]$, un bouton de 3 centimètres de diamètre.

Voici la traduction Precisazioni proposée par Umberto $\mathrm{Eco}^{30}$ :

Alle 12,17 in un autobus della linea S lungo 10 metri, largo 3, alto 3,5, a 3600 metri dal suo capolinea, carico di $\mathbf{4 8}$ persone, un individuo umano di sesso maschile, 27 anni, 3 mesi e 8 giorni, alto 1,62 e pesante 65 chilogrammi, con un cappello (in capo) alto 17 centimetri, la calotta circondata da un nastro di 35 centimetri, interpella un uomo di 48 anni meno tre giorni, altezza 1,68, peso 77 chilogrammi, a mezzo parole 14 la cui enunciazione dura 5 secondi, facenti allusione a spostamenti involontari di questultimo su di un arco di millimetri 15-20. [...]

Centodiciotto minuti più tardi [...], alto 1,70, 57 chilogrammi [...] un bottone d'osso di centimetri 3,5 di diametro.

28. Ibid., pp. 85 et 87.

29. Ibid., p. 26.

30. Ibid., p. 27. 
Si l'on compare le texte source et le texte cible, on remarque aisément que le traducteur italien s'est autorisé de nombreuses modifications et non des moindres. En effet, si l'on regarde les tableaux ci-dessous d'une partie des données du texte, on peut voir que dans certains cas - le premier tableau - Eco a respecté le texte de départ, alors que dans d'autres cas - le deuxième tableauil a changé les chiffres donnés par Queneau.

\begin{tabular}{|c|c|}
\hline $12 \mathrm{~h} 17$ & 12,17 \\
\hline 10 mètres & 10 metri \\
\hline 3,5 & 3,5 \\
\hline 48 personnes & 48 persone \\
\hline 27 ans 3 mois 8 jours & 27 anni, 3 mesi e 8 giorni \\
\hline $65 \mathrm{~kg}$ & 65 chilogrammi \\
\hline 17 centimètres & 17 centimetri \\
\hline 35 centimètres & 35 centimetri \\
\hline
\end{tabular}

\begin{tabular}{|c|c|}
\hline 2,1 & $\underline{3}$ \\
\hline $3 \mathrm{~km} 600$ & $\mathbf{3 6 0 0}$ metri \\
\hline $1 \mathrm{~m} 72$ & $\underline{1,62}$ \\
\hline 48 ans, 4 mois 3 jours & $\underline{48 \text { anni meno tre giorni }}$ \\
\hline 118 & Centodiciotto \\
\hline $71 \mathrm{~kg}$ & 57 chilogrammi \\
\hline 3 centimètres & $\underline{\text { centimetri } 3,5}$ \\
\hline
\end{tabular}

On a surligné en gras dans le deuxième tableau les modifications apportées du point de vue de la forme mais non du contenu puisque la mesure annoncée est respectée mais exprimée autrement. Et on a souligné les données complètement changées.

On peut dorénavant s'intéresser aux formes verbales. Umberto Eco a parfois changé le temps ou la nature de ce qui était dans le texte français mais l'idée évoquée reste plutôt proche de celle de Raymond Queneau :

- Il était chargé : «Carico»

- Était entourée : «Circondata»

- Interpelle : «Interpella »

- Dura (passé) : «Dura » (présent)

- Faisaient : «Facenti »

Enfin, on remarque que pour un même mot écrit en français trois fois dans le texte, Eco peut opter pour trois solutions au lieu de préférer la facilité et n'en garder qu'une. Ainsi, "taille » devient deux fois « alto » et une fois « altezza » et " pesant " " pesante ", " peso " et vient finalement remplacé subjectivement par une virgule :

Taille $\underline{1 \mathrm{~m} 72}$ et pesant $65 \mathrm{~kg}$ : Alto $\underline{1,62}$ e pesante 65 chilogrammi.

Taille $\underline{1 \mathrm{~m} 68}$ et pesant $77 \mathrm{~kg}$ : Altezza $\underline{1,68}$, peso 77 chilogrammi.

Taille $1 \mathrm{~m} 70$ et pesant $71 \mathrm{~kg}$ : Alto 1,70, 57 chilogrammi. 
Les modifications apportées par Eco peuvent sembler anodines mais à travers ces changements de mesures, le deuxième personnage, celui qui est interpelé se trouve être plus grand que le premier. De plus, le premier personnage se trouve être plus lourd que l'ami avec lequel il parle devant la Gare Saint-Lazare, alors que dans le texte de Queneau il est plus léger. Ces modifications n'ont certes aucune importance pour la trame elle-même, mais l'imaginaire du lecteur projettera quant à lui une autre représentation de la scène.

Pourquoi Umberto Eco n'a-t-il changé les données que dans ce cas, défiant la notion de fidélité ? Peut-être pour une meilleure sonorité ? Seul lui pourra nous répondre.

Évoquons à present quelques exercices plus linguistiques que l'on pourrait appeler «exercices de langues». Commençons par Javanais :

Version française Javanais en $\mathbf{V}$ : Unvin jovur vevers mividin suvur unvin vautobobuvus deveu lạva livignnévé essévé, jeveu vapeverççuvus unvin jeveveunovomme vavec unvin lonvong couvou e eve t unvin chạvapooveau envantouvourévé pạvar unvune

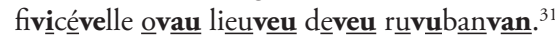

Version italienne Giavanese en F : Ufun giofornofo vefersofo mefezzzofogiofornofo

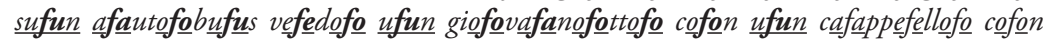

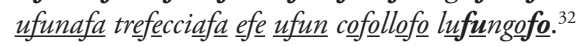

Version espagnole Ticon tila titi en Ti : Tiutina timatina atina tihaticia timetidiotiditia, tien tiel tiautiototibus tide tila tilítinetia tietise tivi tiun ticuetillo timuy tilartigo tiy

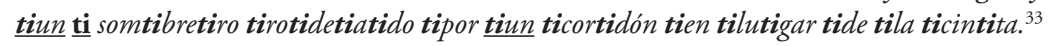

Pour Javanais, on a en français l'insertion de la consonne V suivie de la voyelle ou du son présents dans la syllabe précédente. On remarque dans la version italienne que le traducteur Eco garde une logique linguistique bien qu'il s'agisse d'une sorte de langue inventée puisqu'il accorde le féminin à " ufun » : "ufunafa ». Alors que Queneau insère un V et Eco un F, l'espagnol Fernández Ferrer s'illustre avec la syllabe « $t i$ » entre chaque syllabe du texte source.

Dans le cas de cette variation, Eco a traduit, il n'a pas cherché d'équivalent en italien. Eco ne peut réagir devant Queneau comme le ferait un francophone en France. Il n'a pas les références culturelles nécessaires françaises de base, de même qu'un Français ne connaît pas non plus la culture des enfants italiens. Eco peut ne pas savoir qu'en France les enfants font des chansons avec des langues "inventées ", même si ce mécanisme se retrouve chez les enfants un peu partout dans le monde. Il s'agit de la culture de l'enfance que chacun se crée dès son plus jeune âge.

Dans Javanais et dans d'autres exercices de langue, on remarque une certaine orthonymie dans les procédés utilisés par Queneau qui correspondent au savoir

33. Raymond Queneau, Ejercicios de estilo, op. cit., p. 140. 
moyen des français qui reconnaissent des allusions à des réalités. Les traducteurs ont conservé les styles mais sans qu'il y ait la nécessité sociale de Queneau. Ils ont gardé l'apparence extérieure du texte source.

Analysons maintenant Prosthèses:

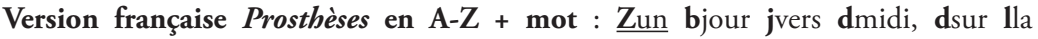
aplateforme zarrière sd'hun tautobus, gnon ploin ddu éparc Omonceaux, èje fremarquai hun éjeune phomme zau pcou strop mlong, cqui sexhibait hun tchapeau centouré d'zun agalon stressé zau mlieu ede truban. ${ }^{34}$

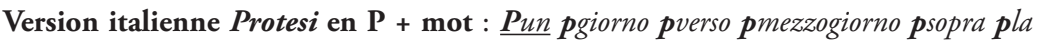
ppiattaforma pposteriore pd'un pautobus pdella plinea PS, pvedo un pgiovane pdal pcollo ptroppo plungo pche pportava un pcappello pcircondato pd'una pcordicella pintrecciata ${ }^{35}$.

Version espagnole Próstesis en A-Z + mot : $\underline{\text { Zuna }}$ bmañana vhacia dmediodía den ela aplataforma ztrasera sde bun tautobús, gno plejos ddel eparque Omonceaux, eobservé fun ejoven, zcon pel pcuello sdemasiado mlargo, cque sexhibia bun tsombrero crodeado dpor zun alagón strenzado zen mlugar ede tcinta ${ }^{36}$.

Pour Prosthèses, on a en français une lettre de l'alphabet suivie du mot, en italien un P suivi du mot et en espagnol une lettre de l'alphabet suivie du mot. Le choix de la lettre en position de préfixe semble aléatoire en français et en espagnol car elle ne suit aucune logique, d'autant plus qu'un même terme est écrit de différentes façons : il suffit pour cela de regarder l'article indéfini dans chaque texte. Quant au " $\mathrm{p}$ » invariable d'Eco, peut-être l'a-t-il choisi pour ne pas avoir à se décider entre toutes les lettres de l'alphabet à chaque début de mot. Il se peut que se soit donc une solution pour «simplifier» la traduction.

\section{Venons-en à Paragoges :}

Version française Paragoges en Mot + A-Z: Ung jourz verse midir, surl laa plateformet arrièreu d'uno autobusi, j'aperçuss uno jeuneu hommeu aux coux tropr longg ett quie portaito ung chapeaux entourée d'ung galong tressés aux lieux deu rubann ${ }^{37}$.

Version italienne Paragoge en Mot + C/K/G... : Unc giornok versoc mezzogiornok soprac lak piattaformak posterioreg di ung autobusb dellac lineak SP vidig ung giovanek dal collok troppok lungog chek portavak ung cappelloc circondatog da unag cordicellam intrecciatam $^{38}$.

Version espagnole Paragoges en Mot + A-Z: Unag mañanaz hacian mediodiar, enl laa platoformat traserau dev uno autobusi, viy uno jovenu coni unt cuellox demasiador largog quen llevabap ung sombrerox rodeadoo porb ung galóng trenzadok enx lugarx deu cintar ${ }^{39}$.

34. Raymond Queneau, Esercizi di stile, Ibid., p. 170.

35. Ibid., p. 171.

36. Raymond Queneau, Ejercicios de estilo, Ibid., p. 132.

37. Raymond Queneau, Esercizi di stile, Ibid., p. 174.

38. Ibid., p. 175.

39. Raymond Queneau, Ejercicios de estilo, Ibid., p. 134. 
Dans Paragoges, en français le mot précède, comme en espagnol, une lettre quelconque de l'alphabet alors qu'Umberto Eco alterne le plus souvent le $\mathrm{C}$, le $\mathrm{K}$ et le G. Certains termes de son texte restent pourtant à leur état pur et il finit la phrase sur une rime inexistante chez Queneau.

Pour Contre-petteries, Queneau commence à jouer avec le lecteur déjà à partir du titre. En effet, le mot s'écrit " contrepèterie " de nos jours mais s'écrivait en deux mots avant que cette nouvelle orthographe ne soit attestée. Umberto Eco propose quant à lui l'orthographe correcte Contre pèteries mais en deux mots alors que le traducteur espagnol opte pour le même titre que Raymond Queneau. Il s'agit dans la version française d'une sorte de métathèse, mais entre deux mots différents et pas au sein d'un même mot.

Ce qui étonne dans l'exercice des Contre-petteries, c'est la plausibilité de ce qui est écrit: en effet, pour une personne ne dominant pas ces langues, les formes et les termes employés pourraient tout à fait sembler exacts.

Dans Anglicismes, on remarque d'importantes interférences linguistiques, l'alternance codique est très importante :

Anglicismes : «Un dai vers middai, je tèque le beusse et je sie un jeugne manne avec une grète nèque et un hatte avec une quainnde de lèsse tressés [...] " ${ }^{40}$.

Anglicismi : "Un dèi, verso middèi, ho takato il bus and ho seen un yungo manno con uno greit necco e un hatto con una ropa texturata [...]" 41 .

Anglicismos : "Un dei a middei, yo teiko el bus y yo sío un yungo manno con un greito necko y un hatto con una queinta leisa trenzados [...] " 42 .

On retrouve plus ou moins les mêmes changements dans les deux versions, mais on remarque par contre une insistance sur le sujet " yo » dans la version espagnole, ce qui n'est pas nécessaire. Cependant, le sujet devant toujours être exprimé en anglais avant le verbe, il est très probable que ce soit une façon d'accentuer l'anglicisme chez Fernández Ferrer.

Dans la version allemande les anglicismes ne sont pas moins nombreux : " dai » (pour " Tag »), " middai » (pour " Mittag »), " teck » (" taken » pour « nehmen »), « kräsie » (pour « verrückt »), « hätt » (pour « Hut »), « läter » (pour « später»), etc.

Dans Italianismes, on peut se demander si le système linguistique proposé par Queneau doit être interprété comme une interlangue ou un interlecte. Ici se pose le problème de la variabilité de l'interlangue : Marina Chini en parle dans Che cos'è la linguistica acquisizionale : "Ci pare [...] opportuna [...] una riflessione sulla variabilità, in quanto la transizione fra uno stadio e l'altro nelle sequenze di apprendimento non è mai netta, ma comprende la possibilità della coesistenza di strutture della fase precedente con strutture "nuove" " ${ }^{43}$.

40. Raymond Queneau, Esercizi di stile, Ibid., p. 168.

41. Ibid., p. 169.

42. Raymond Queneau, Ejercicios de estilo, Ibid., p. 131.

43. Marina Chini, Che cosè la linguistica acquisizionale, Roma, Carocci, 2010, p. 109. 
On a surligné en gras les termes qui semblent révélateurs de l'influence italienne dans le texte de Queneau, et française dans les textes italien et espagnol :

Italianismes : "Oune giorne en pleiné merigge, ié saille sulla plataforme d'oune otobousse et là quel ouome ié vidis? Ié vidis oune djiovanouome au longué col avé de la treccie otour dou cappel $»$.

Francesismi : "Allora, un jorno verso mesojorno egli mi è arrivato di rencontrare su la bagnola de la linea Es un signor molto marante con un cappello tutt'affatto extraordinario, enturato da una fisella in luogo del rubano et un collo molto elongato $" 45$.

Galicismos : "Un jour hacia el midi, él me ha arribada de rencontrar sobre la plataforma arriera de un autobuis de la línea Es un monsieur con un cou trop elongado y un chapeau tutafé extraordinario $" 46$.

Enfin, dans Macaronique, aussi bien le traducteur italien que le traducteur espagnol ont recours au même changement dans certains cas alors qu' ils auraient pu se contenter de laisser le texte tel quel : par exemple en ce qui concerne les formes verbales comme "sudebant ", "portebat "... : elles deviennent "sudabant " et " portabat ». La base verbale reste mais la voyelle thématique change, il s'agit peut-être d'une référence à la forme verbale de chaque langue $(-\mathrm{er} / \mathrm{-ar})$.

Macaronique: «Sol erat in regionem zenithi et calor atmospheri magnissima. Senatus populusque parisiensis sudebant. Autobi passebant completi. In uno ex supradictis autobibus qui $S$ denominationem portebat, hominem a galono tressato cerclato vidi $»^{47}$.

Latino maccheronico : "Sol erat in regionem senithi et calor atmospheri magnissima. Senatus populusque parisensis sudabant. Autobi passabant completi. In uno ex supradictis autobibus qui $S$ denominationem portabat, hominem quasi moscardinum cum collo multo elongato et cul capello a cordincula tressata cerclato vidi ${ }^{48}$.

Latín macarrónico : "Sol erat in regionem zenithi et calor atmospherae magnissima. Senatus populusque parisiensis sudabant. Autobi pasabant completi. In uno ex supradictis autobibus qui $S$ denominationem portabat, hominem quasi juvenum, cum collo molto elongato et cum sombrero a cordincula trenzata circulato vidi " ${ }^{49}$.

Le lecteur comprend le texte dans les trois cas puisque les mots formés sont issus du même lexème (le verbe), en effet la morphologie flexionnelle n'atteint pas la classe à laquelle appartient le mot. En ce qui concerne "passebant » dans le texte source, ou " passabant " dans la version italienne, on peut remarquer l'absence d'un $-s$ en espagnol puisque la prononciation d'une seule consonne

44. Raymond Queneau, Esercizi di stile, op. cit., p. 190.

45. Ibid., p. 191.

46. Raymond Queneau, Ejercicios de estilo, op. cit., p. 144.

47. Raymond Queneau, Esercizi di stile, Ibid., p. 188.

48. Ibid., p. 189.

49. Raymond Queneau, Ejercicios de estilo, Ibid., p. 142. 
provoque le même résultat que deux $-\mathrm{s}$ en français et en italien. Ainsi on a en espagnol " pasabant ". Quant à la dernière phrase, les traducteurs ont choisi différentes solutions, parfois beaucoup plus longues que celle proposée par Raymond Queneau. La version allemande reprend l'intégralité du texte français - pour l'extrait étudié - sauf dans le cas de la fin de la dernière phrase qui conserve cependant " galono tressato cerclato vidi ".

On remarque à travers ces divers exemples que Queneau se fait toujours comprendre par son public, règle qu'a accepté de suivre le traducteur. Pour un francophone les textes sont compréhensibles sans aucun problème. Il suffit de comprendre la règle qui permet de lire l'exercice : lecture phonétique, inversion des lettres, inversion des mots...

Les suites syntagmatiques prennent tout leur sens bien que Queneau ait changé la structure des termes du syntagme ou les unités syntagmatiques au sein même d'un terme. Dans le cas où un terme serait assez transformé, les rapports associatifs permettent au lecteur de retrouver le sens du mot évoqué.

Nous avons donc vu que dans certains cas Queneau joue sur le contenu purement linguistique, et dans d'autres sur la forme - même si ces deux notions sont liées - comme par exemple dans Ode ou Comédie.

Le but premier de Queneau était de proposer une variation de textes à partir d'un premier texte source, en restant toujours en L1 ou en L1 modifiée, mais ne remarque-t-on pas à travers les nombreux exercices une certaine perte d'information et parfois une variation de sens?

Dans certains exercices, il est plus facile de faire passer le style, mais dans d'autres cas que nous avons pu voir, il revient au traducteur de trouver une équivalence dans sa langue ou sa culture.

Les exercices plausibles sont illimités, d'autant plus illimités et intéressants qu'ils ont inspiré d'autres genres d'expression : l'américain Matt Madden a proposé ses propres exercices de style sous la forme d'une bande dessinée. Dans sa préface il tient tout particulièrement à remercier l'auteur français : "The author would like to acknowledge his debt to Raymond Queneau whose influence extends well beyond the inspiration for this book " ${ }^{50}$.

En ce qui concerne les Exercices de style de Queneau, il semblerait qu'ils aient rajeuni sous la plume d'un autre auteur et avec un autre genre. Il est très probable que les traductions vieillissent mais ne peuvent-elles pas aussi rajeunir sous une autre forme?

50. Matt Madden, 99 ways to tell a story. - Exercises in Style, London, Jonathan Cape, 2006, préface. 


\section{Bibliographie}

Chini Marina, Che cos’è la linguistica acquisizionale, Roma, Carocci, 2010, 127 pages. Dotti Ugo, "L'uomo che visse 99 volte ", L'Unità, 8 septembre 1983.

Madden Matt, 99 ways to tell a story. - Exercises in Style, London, Jonathan Cape, 2006, 197 pages.

Queneau Raymond, Ejercicios de estilo, Madrid, Ediciones Cátedra, 2000, 162 pages. Traduction d'Antonio Fernández Ferrer.

Queneau Raymond, Esercizi di stile, Torino, Einaudi, 2008, 319 pages. Traduction d'Umberto Eco.

Queneau Raymond, Exercices de style, Paris, Gallimard, 1979, 176 pages.

Queneau Raymond, Exercices de style accompagnés de 99 Exercices de style typographiques de Robert Massin et de 45 Exercices de style parallèles peints, dessinés ou sculptés de Jacques Carelman, Paris, Gallimard, 1963, 164 pages.

Queneau Raymond, Stilübungen, Frankfurt, Bibliothek Suhrkamp, 1990, 158 pages. Traduction de Ludwig Harig et Eugen Helmlé.

Ragusa Francesco, Raymond Queneau attraverso gli esercizi di stile, Cosenza, Luigi Pellegrini Editore, 1988, 71 pages.

Sanders Carol, Raymond Queneau, Amsterdam/Atlanta, Rodopi, 1994, 111 pages.

Souchier Emmanuel, Raymond Queneau, Limoges, Seuil, "Les Contemporains ", 1991, 303 pages. 